\title{
Unique case of disseminated toxoplasmosis and concurrent hepatic capillariasis in a ring-tailed lemur: first case description
}

\author{
N. Siskos ${ }^{1,2}$, K. Lampe ${ }^{1}$, F.-J. Kaup ${ }^{1}$, and K. Mätz-Rensing ${ }^{1}$ \\ ${ }^{1}$ German Primate Center, Pathology Unit, Göttingen, Germany \\ ${ }^{2}$ Laboratory of Anatomy, Histology and Embryology, School of Veterinary Medicine, Faculty of Health \\ Sciences, Thessaloniki, Greece \\ Correspondence to: K. Mätz-Rensing (kmaetz@dpz.eu)
}

Received: 30 January 2015 - Revised: 2 April 2015 - Accepted: 8 April 2015 - Published: 24 April 2015

\begin{abstract}
A unique co-infection with Toxoplasma gondii and Capillaria hepatica is reported in a semi-freeliving ring-tailed lemur (Lemur catta). In this case acute toxoplasmosis, characterised by severe necrotising inflammation in different organs, was the leading cause of death, whilst accompanying chronic capillariasis was regarded as a predisposing factor. A concise description of both disease manifestations is given.
\end{abstract}

\section{Introduction}

Toxoplasma (T.) gondii, a coccidian parasite with worldwide distribution, can infect nearly all warm-blooded vertebrate species (Tenter et al., 2000). Although infection may be common in many mammalian species, including several nonhuman primate (NHP) species, clinical disease is rare. Interestingly, some species are highly susceptible to infection and often develop fatal toxoplasmosis. These include New World monkeys, prosimians, mountain hares, and Australian marsupials (Dubey et al., 1985; Gustafsson et al., 1997; Juan-Salles et al., 1997). Clinical signs of disease depend on the infected species and the affected organ systems. Highly susceptible species often acquire overwhelming infection and die peracutely without premonitory signs (Dietz et al., 1997). Among primates, lethal toxoplasmosis has been reported mostly in New World monkey species and lemurs; thus both of them seem to be highly susceptible to the disease (Epiphanio et al., 2003; Yabsley et al., 2007).

Hepatic capillariasis is caused by the parasitic metazoan nematode Capillaria $(C$.) hepatica that infects a broad range of primate species (Stidworthy et al., 2009). Featuring a rather simple life cycle, $C$. hepatica requires only one host for development (Brack, 1987). Although rodents are the most frequent hosts, the parasite has been reported in both free-ranging and captive NHP (Pizzi et al., 2008; Stidwor- thy et al., 2009). Despite the fact that deaths due to heavy parasitic burden have been reported, hepatic capillariasis is a mainly silent condition, presenting no actual symptoms, mostly diagnosed post mortem (Pizzi et al., 2008). In this case report, an interesting combination of disseminated toxoplasmosis with $C$. hepatica co-infection in a ring-tailed lemur is presented.

\section{Case history}

An adult male ring-tailed lemur (Lemur catta) from a zoological garden (with access to both indoor and outdoor enclosures) was found dead without any overt signs of disease prior to death. The animal was subsequently submitted to the German Primate Center for necropsy and further investigation. Prominent gross pathologic lesions were found in the small intestine and the mesenteric lymph nodes. Within the jejunum, multifocal ulcerations with hyperaemic margins were present, and the mesenteric lymph nodes were enlarged.

\section{Histology}

The main histologic findings focused on the intestine, the liver, and the lymphatic system. The small intestine presented segmental intestinal necrosis and multifocal, transmural, ulcerative jejunitis. Ulcers were observed only in the je- 


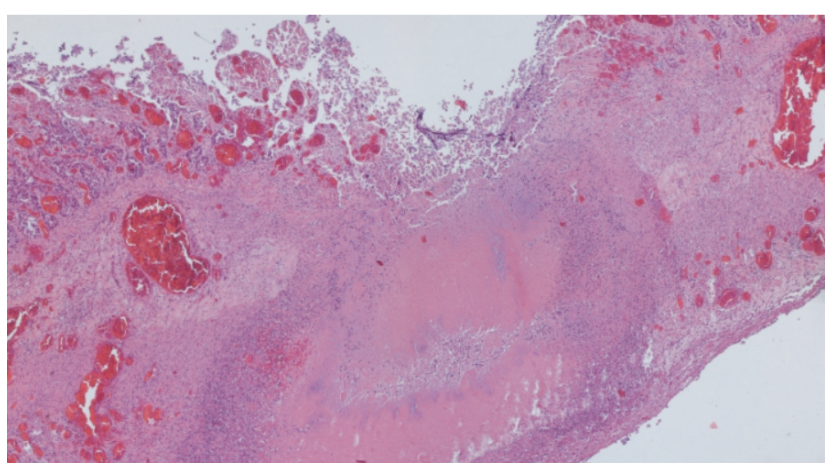

Figure 1. Transmural, ulcerative enteritis, jejunum, ring-tailed lemur (Lemur catta). Transmural ulcer characterised by amorphous, eosinophilic material comprising of cellular debris originating from mucosa, submucosa, and muscularis areas together with inflammatory cell relics. Composition of several low-power (magnification: $\times 5$ ) photomicrographs. Haematoxylin and eosin (H\&E).

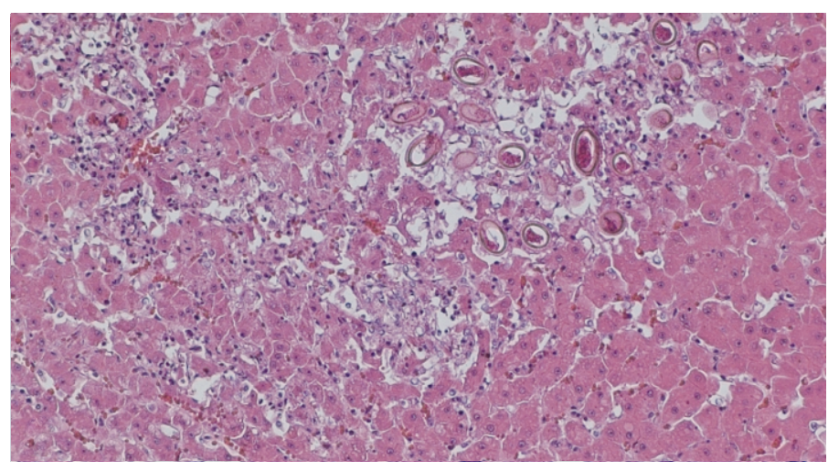

Figure 2. Parasitic, necrotising hepatitis, liver, ring-tailed lemur (Lemur catta). Ovoid structures (sectioned at different planes) represent $C$. hepatica unembryonated bipolar eggs accompanied by multiple foci of hepatocellular necrosis and mild inflammatory cell infiltrate. Magnification: $\times 10$. H\&E.

junum and can be described as transmural but not perforating. Specifically, large eosinophilic areas comprising of cellular debris originating from mucosa, submucosa, and muscularis areas together with inflammatory cell relics substituted the aforementioned layers, leaving the serosa intact (Fig. 1).

Liver histology revealed random, multifocal, necrotising hepatitis. Prominent $C$. hepatica eggs, measuring approximately $52 \mu \mathrm{m}$ long and $30 \mu \mathrm{m}$ wide, featuring an inner refringent and an outer striped wall, were scattered throughout the liver parenchyma (Fig. 2). Pyknosis, karyorrhexis, and cellular debris were indicative of randomly distributed single-cell necrosis, while larger areas were characterised by mixed inflammatory infiltrate consisting primarily of neutrophils, macrophages, and lymphocytes replacing the liver parenchyma (Fig. 3). The presence of fibrotic changes within few necrotic foci, together with subcapsular lymphocytic infiltrate, were both considered features of chronicity. Care-

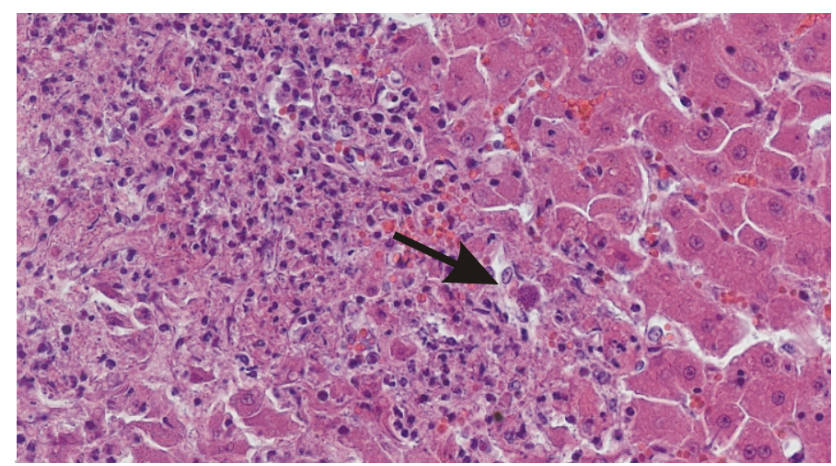

Figure 3. Parasitic, necrotising hepatitis, liver, ring-tailed lemur (Lemur catta). Necrotic focus circumscribed by inflammatory cell infiltrate. A single toxoplasmic tissue cyst is present on the boundaries between healthy and necrotised tissue (arrow). Magnification: $\times 20$. H\&E.

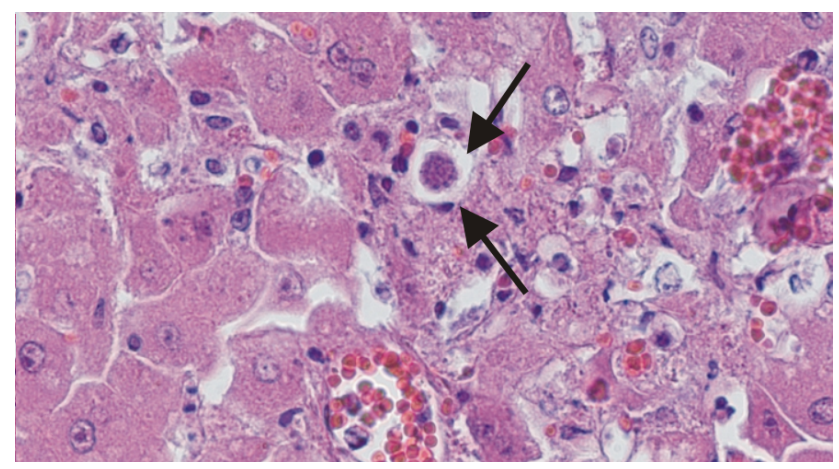

Figure 4. Parasitic, necrotising hepatitis, liver, ring-tailed lemur (Lemur catta). Extracellular T. gondii tissue cyst (arrows) inducing inflammatory response and hepatocellular necrosis. Magnification: $\times 40$. H\&E.

ful, high-power investigation of H\&E-stained liver sections revealed the presence of numerous intra- or extracellular $T$. gondii cysts at the interface between healthy and necrotic tissue (Figs. 3-4). Finally, the simultaneous presence of neutrophils and acute necrotic foci is indicative of acute onset inflammation. Based on these features, it is assumed that $C$. hepatica is the aetiological agent of chronic parasitic inflammation, while $T$. gondii has induced acute protozoal hepatitis.

The spleen and mesenteric lymph nodes were highly lymphocyte-depleted and diffusely necrotic. Splenic white pulp showed neither follicles nor perivascular lymphatic sheaths; cortical follicular architecture in the lymph nodes was also effaced. Furthermore, marked necrosis extended into the splenic red pulp and the medulla of the lymph nodes, while $T$. gondii cysts were frequently observed within the lymphoid-depleted areas. Finally, the central nervous system featured no signs of meningoencephalitis. 


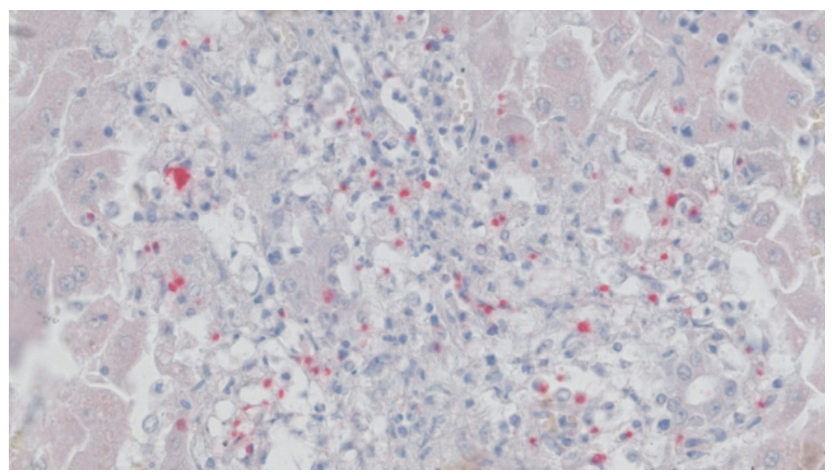

Figure 5. Necrotising hepatitis, liver, ring-tailed lemur (Lemur catta). T. gondii tachyzoites visualised by IHC (red signal), residing in an area of hepatocellular necrosis and prominent inflammatory infiltrate. Magnification: $\times 20$. IHC Toxoplasma gondii.

\section{Immunohistochemistry}

In order to prove the presence of the protozoan, associate it with the lesions, and to study its potential distribution among other organs, a T. gondii-specific immunohistochemistry (IHC) was performed (Figs. 5-6). In more detail, a commercial mouse monoclonal primary antibody against $T$. gondii P30 membrane protein was used (Novocastra, NCLTG) in a $1: 20$ dilution. A biotinylated secondary antibody, streptavidin, and the colour indicator (alkaline phosphatase red detection kit; Ventana, Cat. 760-031) were applied according to the supplier's instructions. Two negative controls were performed. The first included replacement of the primary antibody with sheep serum; the second utilised tissue sections from a healthy animal. Infected tissue served as a positive control. For counterstaining Mayer's haematoxylin was used.

Liver, spleen, lymph node, intestine, pancreas, and lung specimens presented positive signals revealing both the presence of protozoan cysts and/or tachyzoites. While no lesions were noticed in routine histological evaluation, adrenal gland, myocardium, and prostate gland presented minor but nevertheless typical IHC staining consistent with tachyzoites.

\section{Discussion}

The present case confirms the high susceptibility of lemurs to $T$. gondii infection. There have been sporadic reports of deaths in captive ring-tailed lemurs induced by toxoplasmosis (Spencer et al., 2004). In lemurs, and New World monkeys toxoplasmosis leads to acute disease, illness, and death of both host and parasite (synnecrosis), in contrast to Old World monkeys and great apes, which are only minimally affected (parasitism) (Innes, 1997). Attempting to offer an explanation for this difference, some authors state evolutionary reasons. Evolutionary differences in habit and habitat between the definitive hosts and New World monkeys are men-

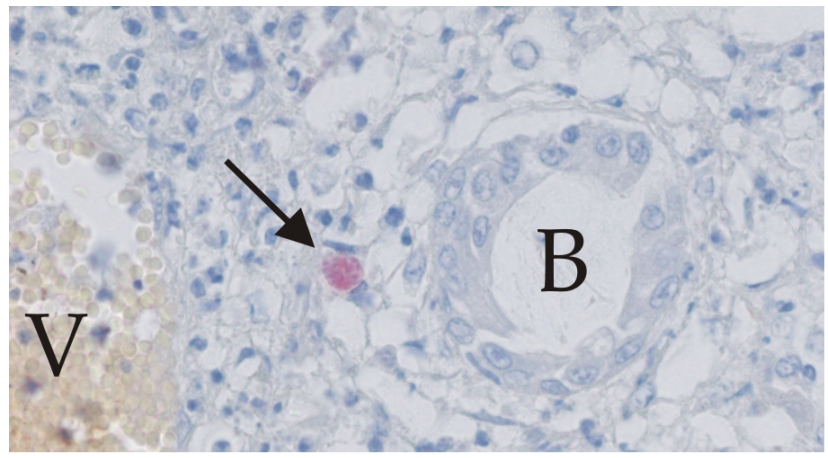

Figure 6. Necrotising hepatitis, liver, ring-tailed lemur (Lemur catta). A single bradyzoite-containing toxoplasmic tissue cyst (arrow) residing between a vein $(\mathrm{V})$ and a bile duct (B). Magnification: $\times 40$. IHC Toxoplasma gondii.

tioned as the main reasons that precluded the latter from developing protective immune responses (Carme et al., 2009; Dietz et al., 1997; Epiphanio et al., 2003; Innes, 1997; Yabsley et al., 2007). According to this hypothesis, the extreme susceptibility may be attributed to the arboreal habits of some New World monkey species and prosimians. Arboreal monkeys are not exposed to oocysts shed with felid faeces to the same extent terrestrial species are. Since parasitism is an evolutionary phenomenon (Epiphanio et al., 2003), arboreal species may have thus failed to develop resistance to toxoplasmosis, in contrast to their non-arboreal counterparts (Carme et al., 2009; Innes, 1997).

Most of the pathomorphologic findings of the present case regarding toxoplasmosis confirmed those already reported for New World monkeys (Brack et al, 1995; Epiphanio et al., 2003). Often there are no gross lesions at necropsy. Pulmonary oedema, congestion, and consolidation may be the only significant necropsy findings. Microscopically, necrotising lesions within liver, lung and heart, accompanied by nonsuppurative meningoencephalitis, are the predominant histologic findings. The distribution of T. gondii organisms in the tissue is variable. Bradyzoites, tachyzoites, and cysts are often widely disseminated, as shown in the present case by immunohistochemistry. Diagnosis is based on demonstration of these organisms in tissue samples by light and electron microscopy. T. gondii tachyzoites are 2-6 $\mu \mathrm{m}$ long, crescent shaped, while cysts are 5-100 $\mu \mathrm{m}$ round thin-walled structures containing few to several hundred bradyzoites (Dubey et al, 1998).

In the present case, diagnosis was confirmed by specific immunohistochemical staining. Immunohistochemical labelling for $T$. gondii antigen was positive in samples of small intestine, liver, spleen, and mesenteric lymph nodes, suggesting the gut as the route of entry for this infection. Furthermore, several other tissues were found to be immunohistochemical positive, which is indicative of systemic infection, despite the variations in the intensity of labelling. 
Acute phase toxoplasmosis features random, multifocal, necrotising hepatitis, directly related to the rapid proliferation of tachyzoites. Chronic capillariasis, which was also diagnosed in this case, provokes hepatocellular necrosis and mild granulomatous response, while granulomatous hepatitis has been recorded only in more severe cases (Pizzi et al., 2009). In this case however, acute disseminated toxoplasmosis is considered to be the actual cause of death. Histological and immunohistochemical analyses emphasise the role of $T$. gondii in the pathogenesis of the aforementioned parasitic hepatitis. Even though the current $C$. hepatica burden is considered too low to be lethal, its contribution requires further investigation. The liver alterations induced by $C$. hepatica may have induced a higher susceptibility for the manifestation of $T$. gondii infection within the organ, leading to severe liver pathology and subsequent spread of the agent to other organs. Based on our own observations in similar cases, concomitant liver hemosiderosis may also act - through the iron overload - as a major predisposing factor for disease development in susceptible species. As suggested by our findings, the liver plays a key role in the pathogenesis, clinical presentation, and pathology of systemic toxoplasmosis. Therefore, there are concerns regarding the substantial influence of chronic subclinical liver alterations in the outcome of the infection.

While the source of the $T$. gondii agents in this case remains undetermined, the most likely scenario is an oral route of infection. Several alterations within the small intestine induced by $T$. gondii support this assumption. Felids are the only known definitive hosts for $T$. gondii, and ingested oocysts shed by cats are the source of infection for several mammalian species acting as intermediate hosts. The role of nondomestic felids in the epidemiology is unknown. Freeroaming house cats defecating near or within the enclosures could serve as a potential source of infection, as they might contaminate stored wood chips intended for bedding materials, or fruits and vegetables prepared for feeding. If possible, it is advisable to keep an appropriate distance between the housings of felids and susceptible species. A strict hygiene practice and a feeding programme without raw meat can further reduce the risk of infection.

Finally, this is the first report of $T$. gondii and $C$. hepatica co-infection in a ring-tailed lemur to our knowledge, underscoring this species' vulnerability to similar infections, along with the need of strict surveillance to avoid similar devastating conditions.

Acknowledgements. We are grateful to W. Henkel, E. Lischka, S. Wienstroth, N. Schminke, and L. Hummel for excellent technical assistance.

The author(s) declared no potential conflicts of interest with respect to the research, authorship, and/or publication of this article.

The authors received no financial support for the research, authorship, and/or publication of this article.
Edited by: A. Lewis

Reviewed by: A. Mejia and three anonymous referees

\section{References}

Brack, M.: Agents transmissible from simians to man, SpringerVerlag, Berlin-Heidelberg, Germany, 324-330, 1987.

Brack M., Wohlsein, P., and Kaup, F.-J.: Toxoplasmosis in nonhuman primates, Verh. ber. Erkrg. Zootiere, 37, 183-187, 1995.

Carme B., Ajzenberg D., Demar M., Simon S., Dardé M. L., Maubert B., and de Thoisy, B.: Outbreaks of toxoplasmosis in a captive breeding colony of squirrel monkeys, Vet. Parasitol., 163, 132-135, doi:10.1016/j.vetpar.2009.04.004, 2009.

Dietz, H. H., Henriksen, P., Bille-Hansen, V., and Henriksen, S. A.: Toxoplasmosis in a colony of New World monkeys, Vet. Parasitol., 68, 299-304, 1997.

Dubey, J. P., Kramer, L. W., and Weisbrode, S. E.: Acute death associated with Toxoplasma gondii in ring-tailed lemursm, J. Am. Vet. Med. Assoc., 187, 1272-1273, 1985.

Dubey, J. P., Lindsay, D. S., and Speer, C. A.: Structures of Toxoplasma gondii Tachyzoites, Bradyzoites and Sporozoites and Biology and Development of Tissue Cysts, Clin. Microbiol. Rev., 11, 267-299, 1998.

Epiphanio, S., Sinhorini, I. L., and Catão-Dias, J. L.: Pathology of Toxoplasmosis in Captive New World Primates, J. Comp. Pathol., 129, 196-204, doi:10.1016/S0021-9975(03)00035-5, 2003.

Gustafsson, K., Wattrang, E., Fossum, C., Heegaard, P. M. H., Lind, P., and Ugla, A.: Toxoplasma gondii infection in the mountain hare (Lepus timidus) and domestic rabbit (Oryctolagus cuniculus), J. Comp. Pathol., 117, 361-369, 1997.

Innes, E. A.: Toxoplasmosis: Comparative species susceptibility and host immune response, Comp. Immunol. Microb., 20, 131138, 1997.

Juan-Salles, C., Lopez, S., Borras, D., Domingo, M., Prats, N., and Fernandez, J.: Disseminated toxoplasmosis in susceptible zoo species - a sporadic disease, Proc. Am. Assoc. Zoo Vet. Annual Conference Proceedings, 227-331, 1997.

Pizzi, R., Gordon, J. C., Flach, E. J., Routh, A. D., Clark, B., and Boardman W. S. J.: Capillaria hepatica (syn Calodium hepaticum) in primates in a zoological collection in the UK, Vet. Rec., 163, 690-691, 2008.

Spencer, J. A., Joiner, K. S., Hilton, C. D., Dubey, J. P., ToivioKinnucan, M., Minc, J. K., and Blagburn, B. L.: Disseminated Toxoplasmosis in a captive ring-tailed lemur (Lemur cata), J. Parasitol., 90, 904-906, doi:10.1645/GE-249R, 2004.

Stidworthy, M. F., Lewis, J. C. M., Masters, N. J., Boardman, S. I., Hopper, J. S., de Linan, F. J., Redprobe, S. P., and Sayers, G.: Capillaria hepatica in primates in zoological collections in the British Isles, Vet. Rec., 164, p. 66, doi:10.1136/vr.164.2.66, 2009.

Tenter, A. M., Heckeroth, A. R., and Weiss, L. M.: Toxoplasma gondii: from animals to humans, Int. J. Parasitol., 30, 1217-1258, 2000.

Yabsley, M. J., Jordan, C. N., Mitchell, S. M., Norton, T. M., and Lindsay D. S.: Seroprevalence of Toxoplasma gondii, Sarcocystis neurona, and Encephalitozoon cuniculi in three species of lemurs from St. Catherines Island, GA, USA, Vet. Parasitol., 144, 28-32, doi:10.1016/j.vetpar.2006.09.020, 2007. 